\title{
Process-based forward numerical ecological modeling for carbonate sedimentary basins.
}

\author{
Roger Clavera-Gispert • Òscar Gratacós · Ana Carmona • Raimon \\ Tolosana-Delgado
}

Received: date / Accepted: date

\begin{abstract}
Nowadays, numerical modeling is a common tool used in the study of sedimentary basins, since it allows to quantify the processes simulated and to determine interactions among them. One of such programs is SIMSAFADIM-CLASTIC, a 3D forward-model processbased code to simulate the sedimentation in a marine basin at a geological time scale. It models the fluid flow, siliciclastic transport and sedimentation, and carbonate production. In this article, we present the last improvements in the carbonate production model, in particular about the usage of Generalized Lotka-Volterra equations that include logistic growth and interaction among species. Logistic growth is constrained by environmental parameters such as water depth, energy of the medium, and depositional profile. The environmental parameters are converted to factors and combined
\end{abstract}

R. Clavera-Gispert

Institute of Geophysics and Geoinformatics, Technische Universität Bergakademie Freiberg

Gustav-Zeuner-Str. 12

09596 Freiberg, Germany

Tel.: +34 934021373

E-mail: roger@clavera.cat

Ò. Gratacós

Dept. de Dinàmica de la Terra i de l'Oceà,

Universitat de Barcelona

Martí i Franquès, s/n

08028 Barcelona, Spain

A. Carmona

Dept. de Dinàmica de la Terra i de l'Oceà,

Universitat de Barcelona

Martí i Franquès, s/n

08028 Barcelona, Spain

R. Tolosana-Delgado

Department of Modelling and Valuation, Helmholtz Institute

Freiberg for Resource Technology

Halsbrueckerstrasse 34

09599 Freiberg, Germany into one single environmental value to model the evolution of species. The interaction among species is quantified using the community matrix that captures the beneficial or detrimental effects of the presence of each species on the other. A theoretical example of a carbonate ramp is computed to show the interaction among carbonate and siliciclastic sediment, the effect of environmental parameters to the modeled species associations, and the interaction among these species associations. The distribution of the modeled species associations in the theoretical example presented is compared with the carbonate Oligocene-Miocene Asmari Formation in Iran and the Miocene Ragusa Platform in Italy.

Keywords Forward-Model · process-based · sedimentary basin $\cdot$ ecological model $\cdot$ carbonate production

\section{Introduction}

Sedimentary carbonates represents $20 \%$ of the sedimentary rock record [31]. They are economically important as oil and gas reservoirs, ore deposits, or as sources of industrial minerals. In addition, the chemistry of the atmosphere and oceans is controlled in part by reactions of carbonate minerals with natural waters and these interactions are important in regulating climate [31]. Some authors consider that all carbonate compounds are directly or indirectly of biological origin [39], other consider some cases such as the whitings in the Bahamas, which are thought to be inorganic [30]. In any case, carbonate sediment has largely a biological origin. Carbonate production is related to seawater chemistry, and it is heavily dependent on local to regional environmental conditions, both spatially and temporally. Light intensity, carbonate saturation, salinity, nutrients, and temperature are the environmental 
variables that mainly control the carbonate production rates $[28,39,45]$. Once produced, carbonate sediment is subject to the same controls as clastic sediments (erosion, transport, and deposition). The interaction of biological activity, environmental parameters, and sedimentary processes results in complex architectural deposition and heterogeneous lithology of sedimentary bodies.

The common approach to study sedimentary basins includes field work, study of boreholes and geophysical data. However, other methods may be useful to complement conventional basin analysis in order to quantify the biological and sedimentological processes, as well as their controlling factors, which are typically not observable in the geological record.

Forward numerical modeling is one of these tools in the study of sedimentary basins. It allows us to experiment directly by playing with different parameters and interactions to reproduce the temporal and spatial evolution of a basin.

During the last decades several process-based forward numerical modeling approaches for carbonate and mixed clastic-carbonate systems have been put forward, including Bosence and Waltham [6], Bice [3], Bosscher and Southam [8], Demicco [17], Granjeon and Joseph [21], Norlund [32], Burgess et al. [12], Hüssner et al. [27], Boylan et al. [9], Warrlich et al. [44], Paterson et al. [34], Cuevas-Castell et al. [16], Hill et al. [25], and Burgess [10]. All these carbonate and mixed carbonatesiliciclastic sedimentary models use a common approximation based on a production rate controlled by environmental parameters.

Carbonate sediment generation is closely related to the organisms that produce or induce its precipitation. Given that these organisms live and compete with each other and among themselves for resources (e.g. space, light, food, and nutrients), an ecological model appears as an appropriate tool to simulate carbonate production dynamics. Such an ecological model for carbonate production, resolved at basin scale for geological time scales, was introduced by Bitzer and Salas $[4,5]$ with the code SIMSAFADIM, and afterwards modified by Gratacós et al. [23,22], Carmona et al. [13], and Clavera-Gispert et al. [15] with the code SIMSAFADIMCLASTIC (SF-CL).

This code is a $3 \mathrm{D}$ process-based forward numerical model to simulate clastic sedimentation and carbonate production, implemented in FORTRAN 95 programming language. The code uses a finite element (FE) method to discretize the modeled basin and solve the equations of the processes considered.

The parameters and processes used in SF-CL are summarized in Fig. 1. The flow, transport and clastic

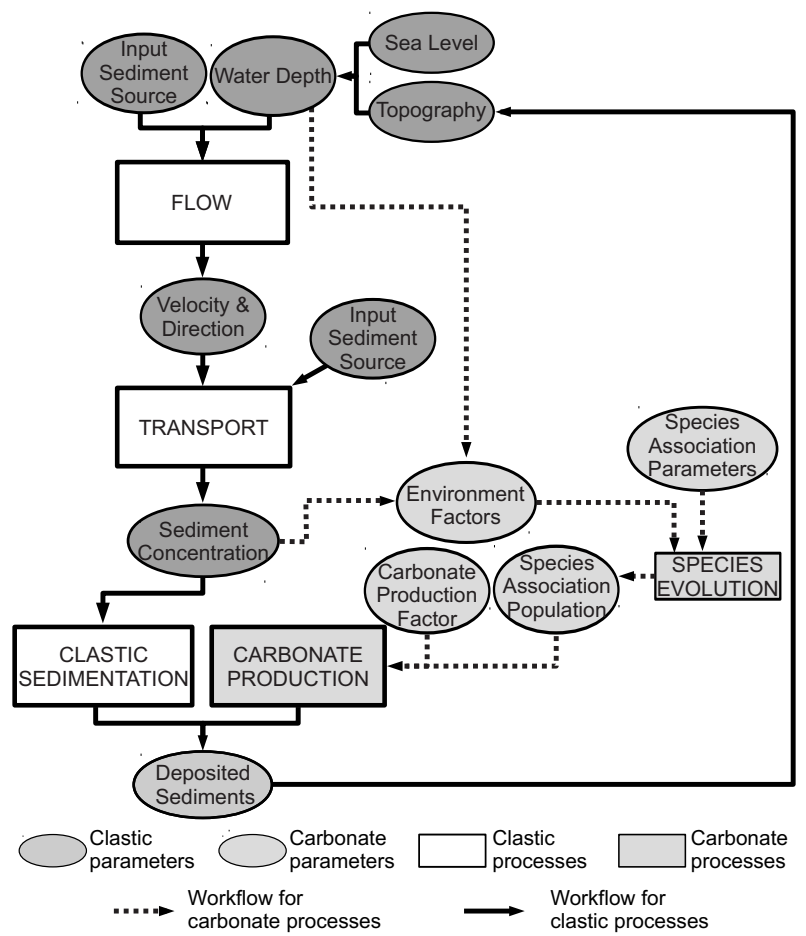

Fig. 1 Schematic diagram of the program.

sedimentation processes are the same used in the previous versions. For more details about these processes and the code in general, the reader is referred to the previous authors.

In this contribution, a new approach for carbonate production using the previous version of SF-CL is presented. The model takes into account the evolution of carbonate producing species as a function of (i) the environment (slope, energy, light), (ii) some intrinsic factors of each species, and (iii) the interaction among them as the sedimentary basin evolves along a geologically-relevant time scale. The implemented model is tested with a theoretical sample experiment. Afterwards, the results of this experiment are compared with two real cases that serve as analogues.

\section{Generalized Lotka-Volterra model.}

The most common models of species evolution in ecological modeling are the predator-prey Lotka-Volterra (LV) equation and its modifications. Previous versions of SF-CL uses the predator-prey equations, and it allowed to model the interaction among 3 species associations only $[4,5]$.

From LV equations, Roberts [36] and Tregonning and Roberts [43] formulated the Generalized LotkaVolterra (GLV) equation (Eq. 1 and 2) that allows unlimited number of species and different types of inter- 
actions among species (Table 1). The GLV equation is mainly formed by two parts, the logistic growth/decay of a species, and its interaction with the other species,

$\frac{d x_{i}}{d t}=\varepsilon_{i} x_{i}+\sum_{j=1}^{N s} \alpha_{i j} x_{i} x_{j}$

where $x_{i}$ is the population density of species $i$; $\varepsilon_{i}$ is the intrinsic rate of increase/decrease of a population of species $i$ (also called Malthusian parameter); $\alpha_{i j}$ is the interaction coefficient among the species association $i$ and $j$, (a particular case is $\alpha_{i i}$, the interaction of one species association with itself), and $t$ is time. Eq. 1 can be written in matrix formulation as

$\frac{d x_{i}}{d t}=\operatorname{diag}[X](\varepsilon+A X)$

where $X$ is the vector of population densities of each species $i, \varepsilon$ is the vector of all Mathusian parameters, $A$ is the matrix of interaction coefficient, also known as community matrix, and $\operatorname{diag}[X]$ is a square matrix with diagonal elements equal to $X$, and zeros outside the diagonal.

The GLV equations (Eq. 1 and 2) do not necessarily correspond to a stable system, i.e. some combinations of $\varepsilon$ and $A$ might correspond to systems that quickly produce the extinction of some or all species associations considered, hence leaving no trace in the geological record. The stability of this system is mostly controlled by the eigenvalues of $A$ [20]. Thus, a species association extinction might be related with changes in this matrix.

\subsection{Logistic equation}

A typical model used for a single species development is the logistic equation (e.g. [39,33,20]), mathematically expressed in Eq. 3 as follows

$\frac{d x}{d t}=\varepsilon x-\varepsilon \frac{x^{2}}{K}$

It relates through time $t$ : the species population $x$; the intrinsic rate $\varepsilon$ of increase of a population; and the carrying capacity $K$, i.e. the maximum number of individuals an habitat can support. Eq. 3 is equivalent to Eq. 1 for a species with $\alpha_{i i}$ equal to $-\varepsilon / K$.

Both variables, $\varepsilon$ and $K$, are determined by intrinsic properties (e.g. birth and mortality), and environmental factors (e.g. light, nutrients, clastic sediments in suspension). Solutions follow curves similar to those shown in Fig. 2.

There are several techniques to determine the values of $\varepsilon$ and $K$ in modern ecosystems, including statistics
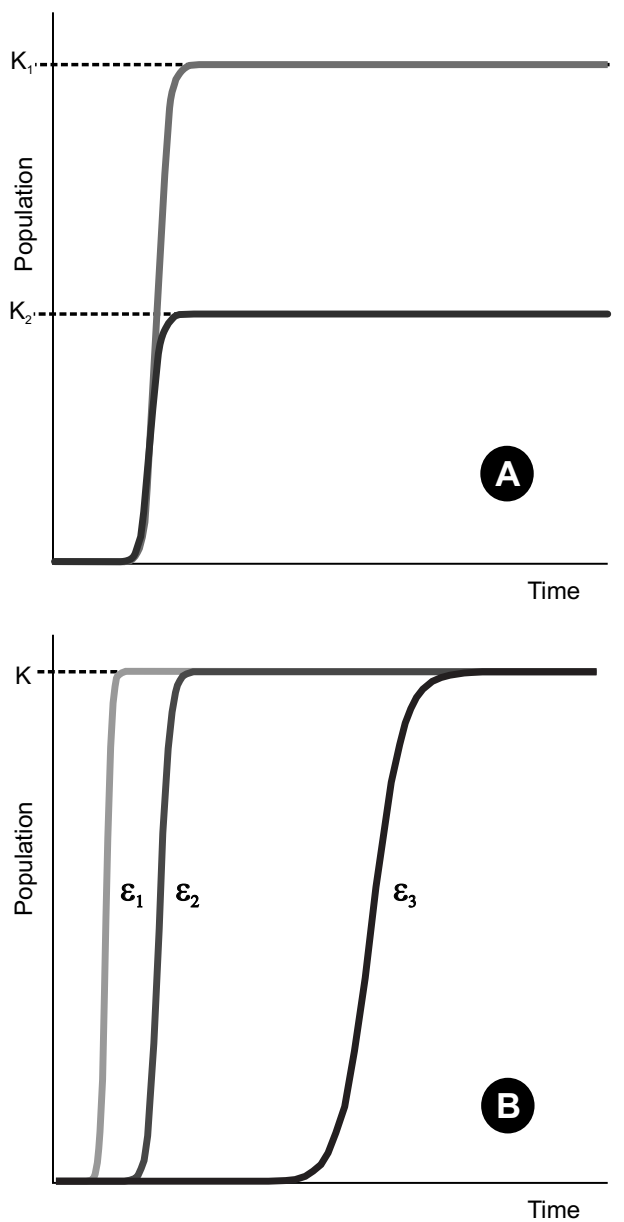

Fig. 2 Sigmoidal growth curves of a species using the logistic equation (Eq. 3). A: Results using two different $K$ values $\left(K_{1}>K_{2}\right)$ and the same value for $\varepsilon$, resulting a greater population in $K_{1}$. B: Results for three different $\varepsilon\left(\varepsilon_{1}>\varepsilon_{2}>\right.$ $\left.\varepsilon_{3}\right)$ and the same $K$ value, obtaining a most rapid creation of niche using $\varepsilon_{1}$ than using $\varepsilon_{3}$.

methods (e.g. [40]), laboratory experiments (e.g. [18]), or estimations from observation (e.g. [19]).

In contrast, these parameters $\varepsilon$ and $K$, can not be deduced from the fossil record by direct observation, neither using laboratory techniques. Thus, only statistics methods for estimating these parameters are possible (applying actualism and deduction from the fossil record). The estimates $\varepsilon$ and $K$ depend on the environmental conditions and the intrinsic characteristics of the species. For example, benthic autotrophic species need access to light for their photosynthetic activity, or feeders need to capture food particles from the water. Thus $K$ could be reasonably assumed to be proportional to the available sea surface. On the other hand, determining possible values for neritic species (like plankton or ammonites) is more difficult.

From a geological perspective, the growth of a species by intrinsic reproduction to its maximum carrying value 
can be reasonably considered to be immediate. Hence we assume $\varepsilon=1$, thus the populations depend only on $K$.

\subsection{Interaction among species.}

The community matrix (introduced in Eq. 2) expresses numerically the relationship among the different species. Individual entries of this matrix are always values between -1 to 1 , defining detriment $(-1)$, benefit (1) or no affection (0) between species. Table 1 shows seven different types of interactions according to possible values of $\alpha_{i j}$. As an illustration of the flexibility of this model, Fig. 3 shows the evolution of five species with different interactions between them.

The community matrix of the LV and GLV equations describe the dynamics of an ecosystem at a time scale and a time resolution that allows to resolve the lifespan of the individuals of each species, whereas the time scale recorded by fossil communities is far larger. Therefore, it might not be possible to compare model results with geological data with regards to which individuals could have been living together in a definite time period and their relationship. Because of this, it is not feasible to estimate the values of interaction coefficients with statistical techniques.

The only plausible way to apply the LV and GLV equations to the geological record is to fix the interaction behaviour using a predation-prey-mutualism-symbiosiscompetition conceptual relationship and ascribe some reasonable values to this qualitative assessment (Table 1). Such quantifications are not verifiable, neither universal, and can only be applied individually to each case study.

\section{Environmental parameters.}

The carbonate production model in SF-CL used as a base model, takes into account the following controlling factors: siliciclastic sediments in suspension, nutrients, and water depth as a proxy for light $[4,5,15]$. In this contribution the following factors are also added: slope; energy of the medium; and light affection.

\subsection{Light.}

Light is one of the most important parameters since many carbonate producers are photoautotrophic organisms. Therefore, light plays an important role controlling carbonate production. The relationship between
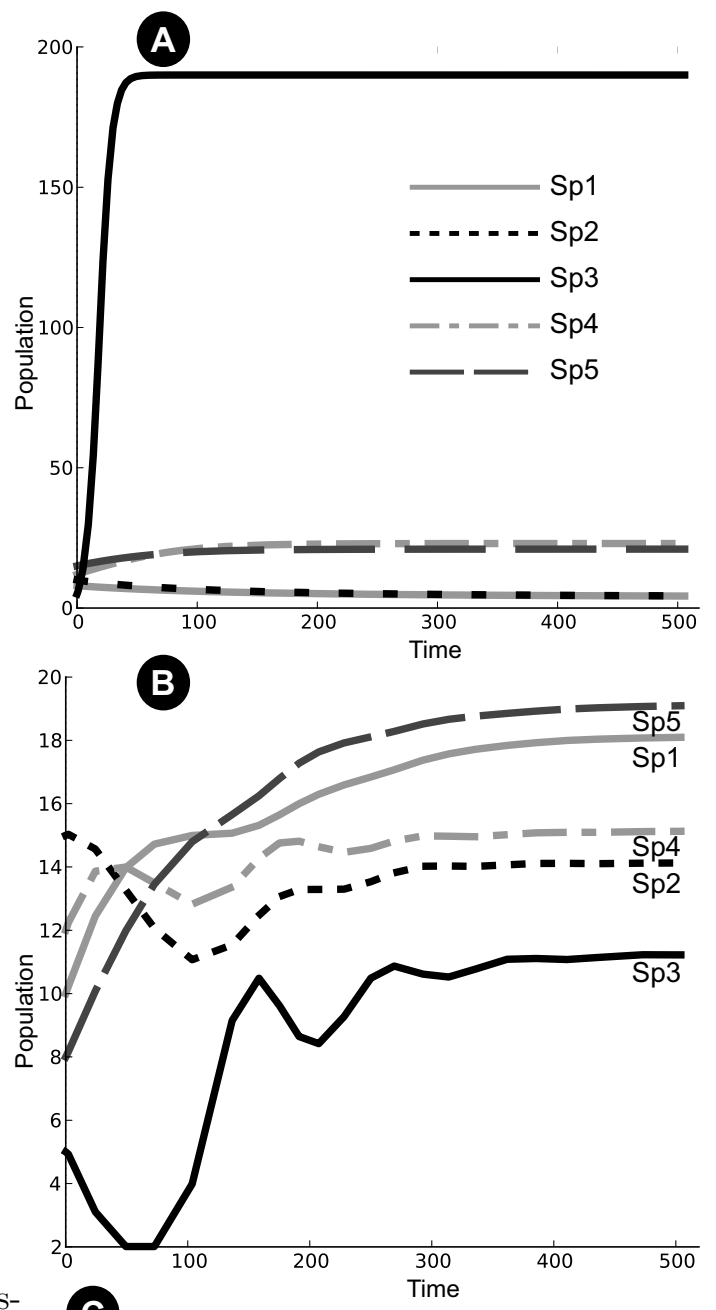

C

\begin{tabular}{cccccc} 
& $\alpha_{\mathrm{i} 1}$ & $\alpha_{\mathrm{i} 2}$ & $\alpha_{\mathrm{i} 3}$ & $\alpha_{\mathrm{i} 4}$ & $\alpha_{\mathrm{i} 5}$ \\
\hline$\alpha_{1 \mathrm{j}}-0.001$ & 0.001 & 0.0 & 0.0 & 0.0 \\
\hline$\alpha_{2 \mathrm{j}}-0.001$ & -0.001 & 0.001 & 0.0 & 0.0 \\
\hline$\alpha_{3 \mathrm{j}}$ & $0.0034-0.001$ & -0.001 & -0.02 & 0.004 \\
\hline$\alpha_{4 \mathrm{j}}$ & 0.0 & 0.0 & 0.001 & -0.001 & -0.001 \\
\hline$\alpha_{5 \mathrm{j}}$ & 0.0 & 0.0 & 0.0 & 0.001 & -0.001 \\
\hline
\end{tabular}

Fig. 3 Graphical evolution of 5 species using the GLV equations. A. Evolution without species interaction. Note the typical evolution of the logistic equation. B. Evolution of the 5 species using the interactions defined by the community matrix C.

carbonate production, photosynthesis and light is evidenced by the decrease of carbonate production with water depth [39].

Common current numerical models take carbonate production rate to primarily and strongly depend on depth. Analytical forms to model this dependence are obtained by relating carbonate sedimentation to known exponential function for light attenuation in the ocean, typically for coral growth and, consequently, for shallow water carbonate production $[3,35,7,8,27,5,9,44,16,25]$.

The original carbonate production module $[4,5]$ for shallow water has been extended to include all possi- 
Table 1 List of interaction among species, the effects on species, and rang of $\alpha_{i j}$ values.

\begin{tabular}{ccccc}
\hline Interaction & Effects on $i$ & $\alpha_{i j}$ range & Effects on $j$ & $\alpha_{j i}$ range \\
\hline Neutralism & no affection & $\alpha_{i j}=0$ & no affection & $\alpha_{j i}=0$ \\
Amensalism & detrimental & $-1 \leq \alpha_{i j}<0$ & no affection & $\alpha_{j i}=0$ \\
Commensalism & beneficial & $0<\alpha_{i j} \leq 1$ & no affection & $\alpha_{j i}=0$ \\
Competition & detrimental & $-1 \leq \alpha_{i j}<0$ & detrimental & $-1 \leq \alpha_{j i}<0$ \\
Mutualism & beneficial & $0<\alpha_{i j} \leq 1$ & beneficial & $0<\alpha_{j i} \leq 1$ \\
Predation & beneficial & $0<\alpha_{i j} \leq 1$ & detrimental & $-1 \leq \alpha_{j i}<0$ \\
Prey & detrimental & $-1 \leq \alpha_{i j}<0$ & beneficial & $0<\alpha_{j i} \leq 1$ \\
\hline
\end{tabular}

ble marine carbonate production systems, defined by means of influence curves, which the user can flexibly constrain (detailed in section 3.4).

\subsection{Energy of the medium.}

Energy of the medium is a local and regional parameter controlling growth of carbonate producing organisms. Nonethless, in certain cases such as for coral reefs, it has been suggested to have a much more important controlling effect at a regional scale [28]. For example, wave energy determines the morphology and growth rates of carbonate-producing organisms; e.g., the coral branching complexity decreases as hydrodynamic stress increases [14].

Several authors include this parameter to control the carbonate production, including Bosscher and Southam [8], Demicco [17], Granjeon and Joseph [21], Nordlund [32], and Burgess and Emery [11].

SF-CL includes two parameters to simulate the effects of energy of the medium. The first one is a wave baseline, above which no sedimentation occurs. The second one is a parameter as a function of flow velocity, which in turn depends on water depth and distance from the input point. A piece-wise linear curve forming a trapezoid (Fig. 4) can be specified as an input parameter in the code to control the effect of this factor on each species growth.

\subsection{Slope.}

The depositional profile is another factor controlling carbonate producing species. For example, in steep shores, waves bounce back without reducing their energy, whereas mildly sloping shores dissipate all the waves energy without bouncing them back. A flat surface or a gently sloping sea bottom faces the sunlight better and gets an even amount of light from morning to evening, while steep walls may never face the sunlight or receive it for only short periods of the day. Several authors including Hubbard and Scaturo [26],
Letourneur et al. [29], and Roff et al. [37] take this factor into account in the study of present ecosystems.

Up to the authors knowledge, the slope of the bottom surface is not included explicitly as a controlling parameter in any other forward numerical models applied at geological time scale. SF-CL computes the slope of the bottom topography in each element of the finite elements mesh and includes this parameter as an environment factor for carbonate production using the standard trapezoidal function detailed in the next section.

3.4 Combining environmental parameters and carbonate production.

SF-CL implements an influence function for each environmental factor (water depth, slope, fluid flow, and nutrients), plus a function for each siliciclastic sediment type in order to model the interaction with carbonate producing organisms. For the sake of simplicity, these functions have a trapezoidal shape that the user can define through 4 points: a minimum value, a maximum value, and 2 optimal values as shown in Fig. 4 . The function is linearly interpolated between these points. Thus, below the minimum (A) and above the maximum (D) no production can occur (influence=0). Between the two optimal values ( $\mathrm{B}$ and $\mathrm{C}$ ), production is considered unhindered by this factor (influence=1). Finally between the minimum (A) and the first optimal point (B), or between the second optimal point (C) and the maximum (D), the influence is linearly interpolated.

All these functions return influence values between 0.0 and 1.0, that are combined into one single environmental hindrance value using one of the following two ways: through the rule of the minimum,

$f_{\text {env }}=\min \left\{f_{\text {flow }}, f_{w d}, f_{\text {nutr }}, f_{\text {clst } s}, f_{\text {slp }}\right\}$

or through the multiplicative rule,

$f_{\text {env }}=f_{\text {flow }} f_{w d} f_{\text {nutr }} f_{\text {slp }} \prod_{s=1}^{N_{\text {sed }}} f_{\text {clst } s}$ 


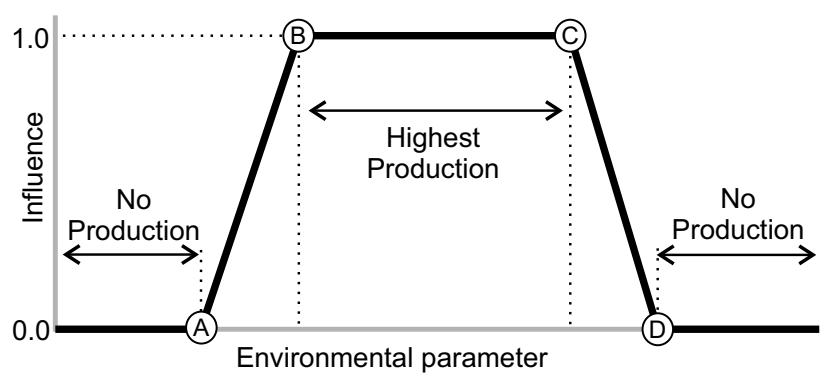

Fig. 4 Trapezoidal function used to compute the influence of each environmental factor (slope, water depth, and fluid flow). This function is defined by 4 points: $\mathrm{A}$ is the minimal value below which the species cannot live. Points $\mathrm{B}$ and $\mathrm{C}$ define the range where the species has the best conditions for development. $\mathrm{D}$ is the value over which the species cannot live either.

where $f_{e n v}$ is the environmental hindrance global factor; $f_{\text {flow }}$ is the effect of fluid energy; $f_{w d}$ is the effect of water depth; $f_{\text {nutr }}$ is the effect of nutrient concentration; $f_{\text {clst } s}$ is the hindrance effect due to presence of siliciclastic sediment class $s ; f_{s l p}$ is the effect of terrain slope, and $N_{\text {sed }}$ is the number of modeled siliciclastic sediments.

The environmental curves of many extinct species are not known, and the information that can be extracted from the geological record is obviously limited. Thereby, the quantification of these parameters is not an easy task. The best way to compute the global environmental factor depends on the availability and the accuracy of these data. Usually, for a species with a wellconstrained environmental sensitivity to each of these factors, the multiplicative rule appears to be the best option. On the other hand, the rule of the minimum is more robust, thus it will be more appropriate for a species which environmental sensitivity is only roughly known.

The effect of choosing the minimum rule or the multiplicative rule can be seen graphically in a synthetic example in Fig. 5.

In the current implementation, this global environmental factor downscales the intrinsic rate of increase of a population $\varepsilon$ of Eq. 1 as:

$\varepsilon_{i}=\varepsilon_{\max i} f_{\text {env }}$

where $\varepsilon_{\max i}$ is the maximum growth rate of species association $i$ at the optimal environmental conditions.

Once a species association population is computed, carbonate production is calculated using a carbonate production factor. Production factors are specified for the maximum population, and linearly scaled to the actual population following the relation

$\frac{d P}{d t}=R_{\max } \frac{x_{i}}{K_{i}}$
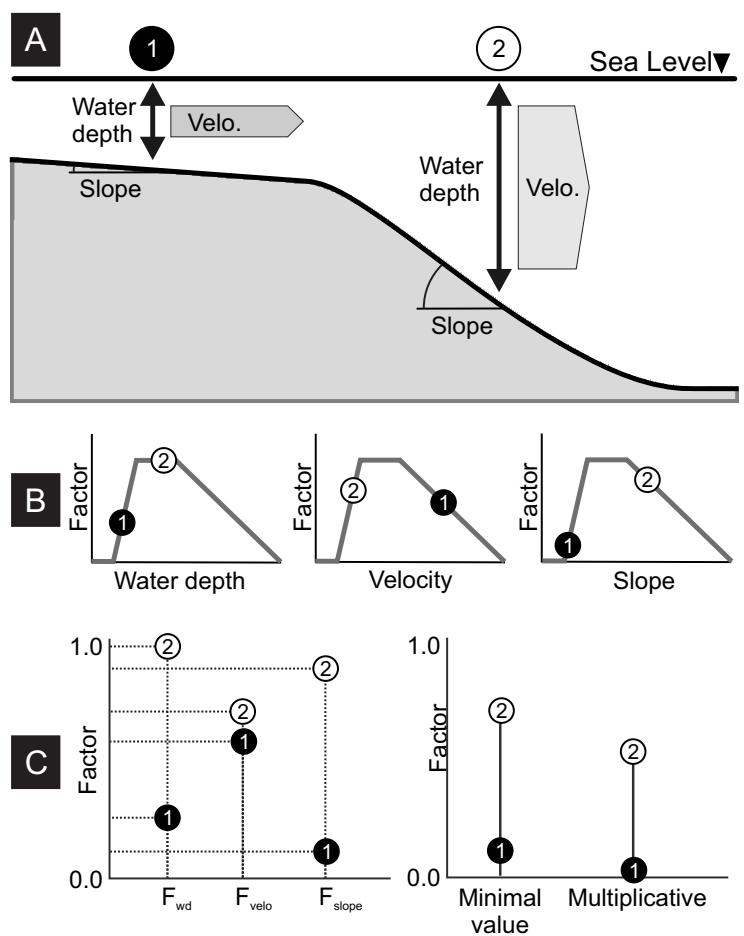

Fig. 5 Conceptual model of a basin and the environmental parameters used to computing the species susceptibility to environmental conditions. A. An idealized basin with the environmental conditions (water depth, flow velocity and slope) used to model the species evolution for the species 1 and 2 . B. Functions to quantify the affection of each specie under these environmental conditions. C. Environmental factors and combination of these parameters using both rules, the multiplicative and minimum value.

where $P$ is the carbonate production; $t$ is time; $R_{\max }$ is the carbonate production factor when population is at its maximum; and $K_{i}$ is the maximum population of species $i$, computed as:

$K_{i}=\frac{\varepsilon_{i}}{\alpha_{i i}}$

\subsection{Numerical method}

The conceptual and methematical model for the carbonate production results in a set of differential equations (ODEs), one for each species associations modeled. The Runge-Kutta-Fehlberg method (RKF45) is used to solve this GLV ODEs system. This method is selected due to it is an explicit method with a stepsize control and dense output data. Similar methods has been tested, such as Runge-Kutta of order $8(5,3)$, but they are slower than the chosen method. Considering that the GLV ODEs equations are 1D, they do not explicitly depend on spatial coordinates and can be solved at each node of the FE mesh.

The RKF45 method requires 4 parameters to solve the GLV ODEs: 
- the step-size (or time step) that is a parameter obtained automatically by the program. The program discretizes the total modelling time (defined by the user as an input parameter) in several time steps in order to solve the equations system. The discretization is done according to the Courant stability criterion in order to avoid numerical errors [41]. This criteria can be obtained in function of the faster process in the basin and ensures that a sedimentary particle can be transported from one node of the FE mesh to the next one within a time step. The time step is obtained from the fluid flow velocity and the spatial discretization of the FE mesh. Thus, it is assumed that within a time step, all the modelled geological processes remains constant $[5,22]$.

- initial population of the species association. This is an input parameter initially defined by the user as an initial condition. This value is used by the program to obtain the population at the end of the first time step. This population is then used as an input parameter for the next time step and so on.

- tolerance that refers to the maximum error that is accepted for the equation system solution.

- and the safety factor that ensures that the solution is within the tolerance[24]. This parameter together with the previous one are defined by the user as input parameters and both are related to the solution quality.

Finally, the representative population and carbonate production are obtained at each time step and for each node of the FE mesh, .

\section{Synthetic sample experiment.}

\subsection{Initial set-up.}

A theoretical experiment has been used to test the new capabilities of the improved carbonate production model. This example models a carbonate ramp of 24.01 $\mathrm{km}^{2}(4900 \mathrm{~m} \times 4900 \mathrm{~m})$ discretized into 50 columns and 50 rows, obtaining a mesh with 2500 nodes and 4802 elements, as displayed in Fig. 6A. Initial submarine basin topography defines a ramp ranging from 0.0 $\mathrm{m}$ at its northern side to a maximum of $150.0 \mathrm{~m}$ at the southern one, resulting a constant sloping surface, with a $2^{\circ}$ dipping angle (Fig. 6). Total simulation time is 90 000 years, divided into 180 time steps of 500 years.

The sea-level position has been initially defined at -35 $\mathrm{m}$, and the sea-level changes combine a sinusoidal function and a linear trend (Fig. 6C):

$S L=-35+30 \sin \left(\frac{2 \pi t}{45000}\right)+25 t$

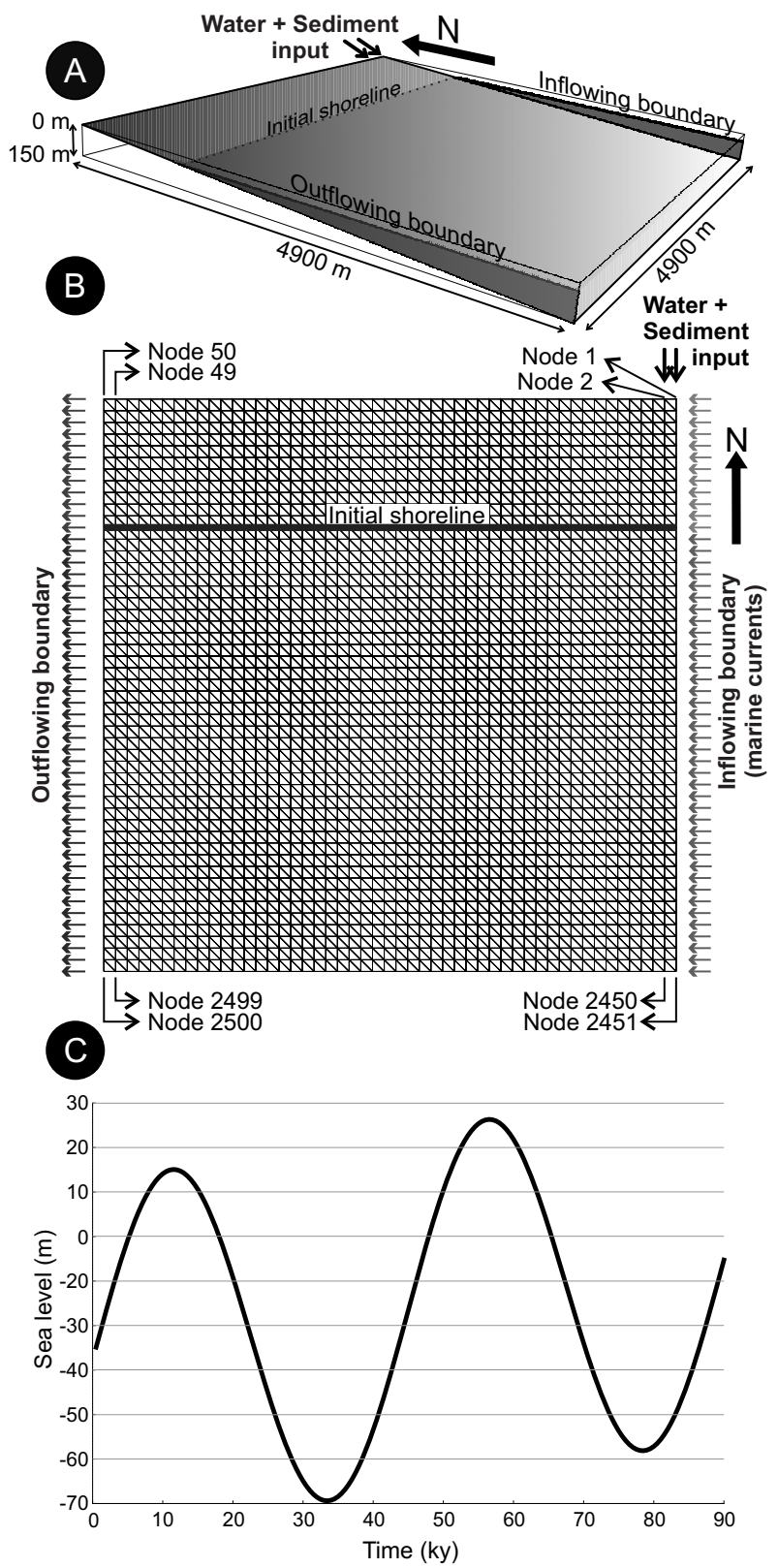

Fig. 6 Experiment set-up. A. 3D view of the initial basin topography and boundary conditions for sediment and water. B. Corresponding finite element (FE) mesh. Right boundary nodes are defined as inflowing nodes, while the left boundary nodes are defined as the outflowing nodes in order to induce E-W marine currents. River discharge is defined through two input nodes in the NE corner. C. sea-level function used to simulate the sea-level changes.

Under these conditions, two main eustatic cycles are obtained (Fig. 6C), trying to force coastline and river discharge migrations and to obtain different depositional systems. This is intended to study the different sedimentary architectures and the effects of coastline migration on carbonate deposits.

Considering this initial set-up, this example has been executed in a Dell ${ }^{\circledR}$ T7610 workstation with Red Hat ${ }^{\circledR}$ 
Linux $^{\circledR}$ 6.5, with 32 Gb RAM and with two Intel ${ }^{\circledR}$ Xeon E5-2687w (3,1Ghz) processors (16 cores, 32 threads). Total runtime for this example has been about 13 hours.

\subsection{Initial and boundary conditions}

Flow model

River inflowing nodes has been defined through two input nodes in the NE corner. Additionally, to induce an E-W marine currents, 50 inflowing nodes are defined in the eastern boundary of the FE mesh, and 50 outflowing nodes in the western boundary (Fig. 6B). This boundary conditions can change depending on the coastline position due to sea-level variations through time.

The obtained fluid flow is represented in Fig. 7 at four different time steps of the simulation time. Independently of the coast line position, it can be appreciated that the fluid flow behaves according to the source point in the NE corner, but it also reproduces a general E-W marine current trend, parallel to the coast line. The maximum velocity is located near the river inflowing nodes, close to the coast line mostly with a NE-SW component depending on where the coast line is located and the sea-level variations. The lowest fluid flow velocities values are located in the Eastern boundary. The values of the fluid flow depend mainly on water depth and the distance from the fluid source.

\section{Siliciclastic transport and sedimentation model}

Initial conditions for sediment transport and sedimentation are defined considering that the basin has no sediment concentration in suspension at time $t=0$ years. Additionally, two grades of siliciclastic sediment (a coarse and a fine) are introduced into the basin through the same two inflowing nodes at the NE corner in order to simulate the river discharge. Each sediment type has been defined using the parameters summarized in Table 2, which control the sediment input and the proportion of each sediment type that is deposited or rest in suspension for transport at each time step, according to its grain size and the fluid flow velocity.

\section{Carbonate production model}

Regarding to the carbonate production model, four species associations have been considered: scleractinian corals, benthic foraminifera, rhodoliths, and planktonic foraminifera. The parameters used and obtained from the bibliography to describe the optimal and suboptimal environments where the different species associations can live are described below and combined using the minimal value rule (summarized in Table 3 ).

Scleractinian corals are common carbonate producers in clear and warm tropical to subtropical shallow waters with moderate energy environment. Thus, in this sample experiment, the optimum water depth where corals can live has been defined between 2 and $20 \mathrm{~m}$, with a maximum of $50 \mathrm{~m}$, the slope of the bottom with low values (maximum of $2.5^{\circ}$ ), and fluid flow velocity ranging from 1 to $40 \mathrm{~m} / \mathrm{d}$.

Benthic foraminifera live in water depths from $1 \mathrm{~m}$ until $200 \mathrm{~m}$ with higher populations between 10 and $40 \mathrm{~m}$, depending on species environment and age [2]. In this example, the maximum depth where this species can live, has been fixed to $165 \mathrm{~m}$, and the optimal values ranging between 10 and $40 \mathrm{~m}$. Moreover, benthic foraminifera are not slope-depending and can live under high energetic conditions.

Rhodoliths live in low intertidal zones to below $150 \mathrm{~m}$, typically in areas where light is strong enough for fostering growth. The range used in the example is between 5 to $150 \mathrm{~m}$. Water motion needs to be strong enough to inhibit sediment burial but not so energetic or unidirectional to cause mechanical destruction or rapid transport out of favourable growing conditions [42]. Thus, optimal energy conditions are defined between 1.5 and $40 \mathrm{~m} / \mathrm{d}$.

Planktonic foraminifera live suspended in seawater column, hence the slope of the bottom profile is an irrelevant factor. Water depth is also not relevant but a range from 0 to $160 \mathrm{~m}$ has been considered for this species. Currents can move this species association out from high fluid flow areas, thus, lower fluid flow velocities needs to be considered. In the example, a range between 0 to $3 \mathrm{~m} / \mathrm{d}$ has been used.

The interaction among species associations is established using the interaction coefficients, defined in the community matrix shown in Table 4 . The values used force a no-interaction scenario $\left(\alpha_{i j}=0.0\right)$ when the two species live in different range of water depth, flow velocities, or slope. The values of $\alpha_{i j}<0$ define competition between species for resources (e.g. space, light) because all species are photosynthetic species without any predator-prey relationship between them, in the example, the values used indicate low interaction. The internal competition is defined in all species associations as $\alpha_{i i}=-0.01$ indicating low internal competition.

\subsection{Results and discussion}

Siliciclastic sediment distribution 

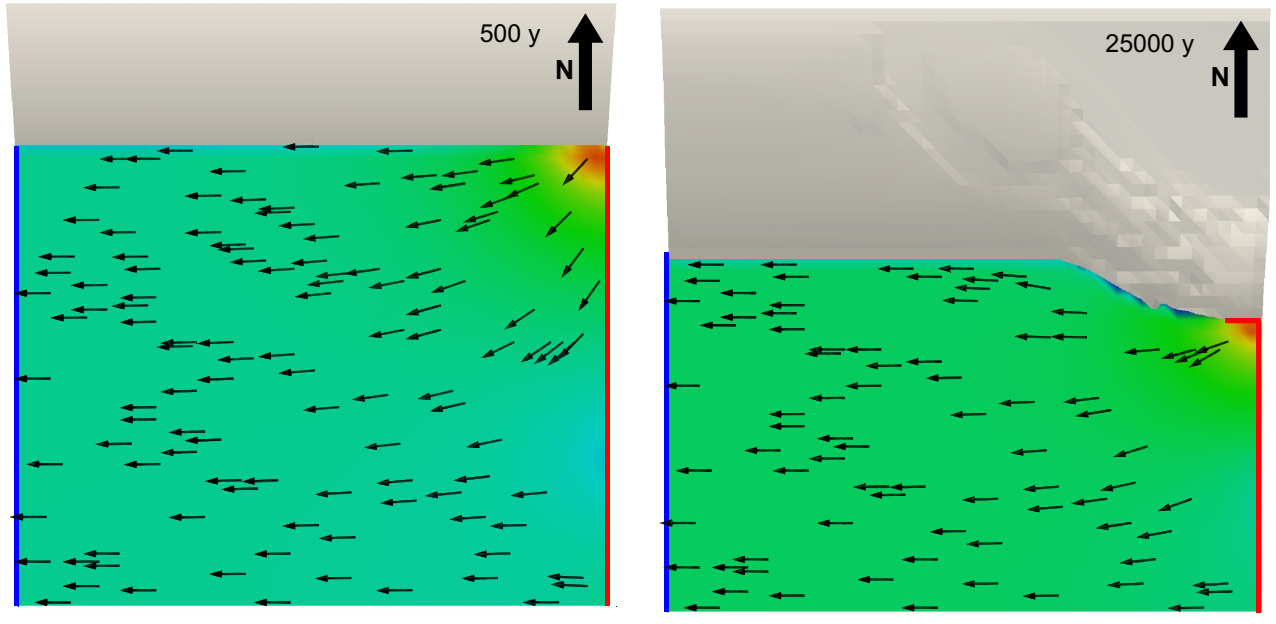

0.1

10

$100 \quad 250$

Fluid flow velocity $(\mathrm{m} / \mathrm{d})$
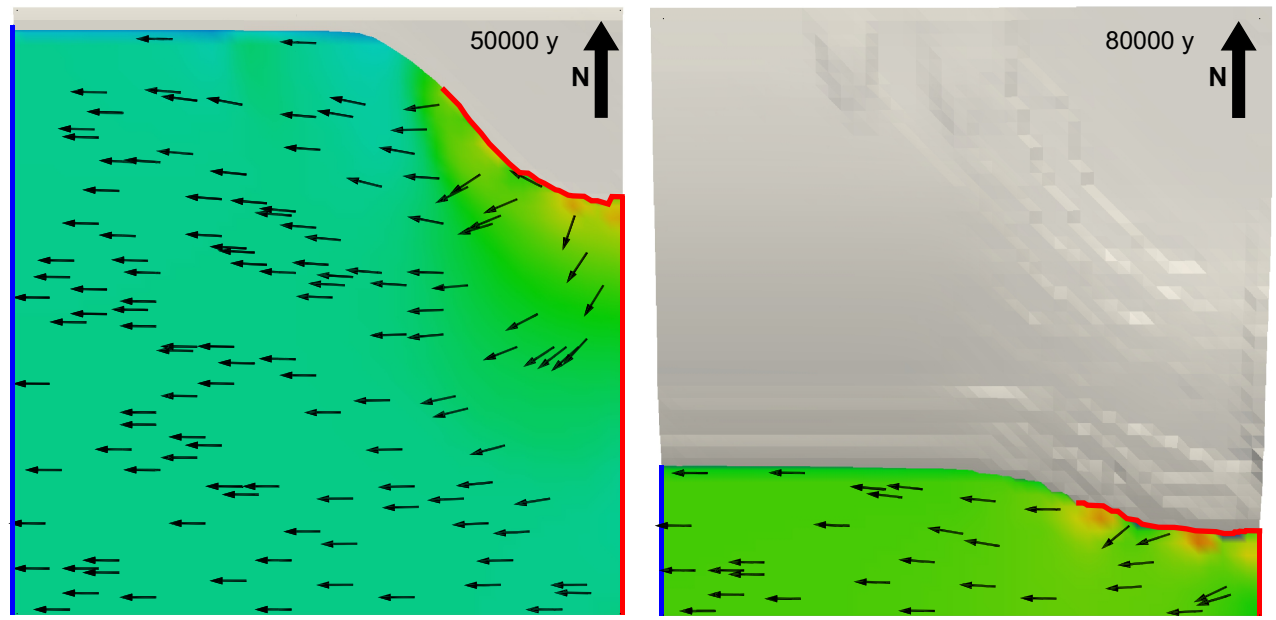

Fig. 7 Fluid flow computed at 500, 25000, 50000, 80000 years. Note the colour scale is logarithmic, and the fluid flow direction arrows are represented at a random sample locations. Red line indicates the inflowing boundary and blue line marks the outflowing boundary.

Table 2 Parameters used to define the two siliciclastic sediments in the example. Following [22], maximum flow for deposition is a critical value below which sediment can be deposited (as a function of the settling and fluid flow velocity). Longitudinal and Transversal dispersivity are defined as a function of the finite element mesh discretization in order to avoid numerical errors solving the transport equation. In turn, the finite element mesh is defined as a function of the expected heterogeneity.

\begin{tabular}{ccccccccc}
\hline & $\begin{array}{c}\text { Input } \\
\text { nodes }\end{array}$ & $\begin{array}{c}\text { Sediment } \\
\text { input } \\
\left(\mathrm{T} / \mathrm{m}^{3}\right)\end{array}$ & $\begin{array}{c}\text { Settling } \\
\text { rate } \\
(\mathrm{m} / \mathrm{d})\end{array}$ & $\begin{array}{c}\text { Max.flow } \\
\text { for deposition } \\
(\mathrm{m} / \mathrm{d})\end{array}$ & $\begin{array}{c}\text { Density } \\
\left(\mathrm{g} / \mathrm{cm}^{3}\right)\end{array}$ & $\begin{array}{c}\text { Longitudinal } \\
\text { dispersion } \\
\left(\mathrm{m}^{-1}\right)\end{array}$ & $\begin{array}{c}\text { Transversal } \\
\text { dispersion } \\
\left(\mathrm{m}^{-1}\right)\end{array}$ & $\begin{array}{c}\text { Diffusion } \\
\left(\mathrm{m}^{2} / \mathrm{s}\right)\end{array}$ \\
\hline Coarse siliciclastic & 1 and 51 & 0.0006 & 1.06 & 155.0 & 2.7 & 100.0 & 100.0 & $10-7$ \\
Fine siliciclastic & 1 and 51 & 0.002 & 0.005 & 40.2 & 2.7 & 100.0 & 100.0 & $10-6$ \\
\hline
\end{tabular}

In the model, terrigenous sedimentation occurs mainly in the NE area near the defined inflowing nodes (Fig. 8 ). The deltaic systems display different progradationalaggradational-retrogradational patterns that well represent the defined sea-level variations and the corresponding input nodes migration. This relationship causes a complex pattern of facies interfingering and facies het- erogeneity in 3D. As expected, coarse sediments are restricted to proximal areas near the input nodes and fine-grained sediments are deposited basinward. In proportion, deltaic systems are mainly built up by the finest sediment. The sedimentary bodies show typical sigmoidal geometries and stratigraphic architectures in accordance with the basin geometry, sea-level varia- 
Table 3 The four defining points for the trapezoidal functions (Fig. 4) used in the synthetic sample experiment.

\begin{tabular}{cccccc}
\hline & & Min. & Opt.1 & Opt.2 & Max. \\
\hline water depth & Corals & 1 & 2 & 20 & 50 \\
$(\mathrm{~m})$ & Bent.foram. & 1 & 10 & 40 & 165 \\
& Rhodo. & 5 & 50 & 70 & 150 \\
& Pl.foram. & 1 & 50 & 160 & 200 \\
\hline slope & Corals & 0 & 0 & 2.5 & 2.5 \\
$\left(^{\circ}\right)$ & Bent.foram. & 0 & 0 & 89 & 90 \\
& Rhodo. & 0 & 0 & 4 & 15 \\
& Pl.foram. & 0 & 0 & 89 & 90 \\
\hline fluid flow & Corals & 1 & 1 & 39 & 40 \\
$(\mathrm{~m} / \mathrm{d})$ & Bent.foram. & 0 & 0 & 39 & 40 \\
& Rhodo. & 1.5 & 1.5 & 39 & 40 \\
& Pl.foram. & 0 & 0 & 2 & 3 \\
\hline
\end{tabular}

Table 4 Community matrix used in the theoretical example in order to define the interaction among species.

\begin{tabular}{ccccc}
\hline & Corals & Ben.foram. & Rhodo. & Pl.foram. \\
\hline Corals & -0.01 & -0.001 & -0.002 & 0.0 \\
Bent.foram. & -0.001 & -0.01 & -0.001 & 0.0 \\
Rhodo. & -0.002 & -0.01 & -0.01 & -0.001 \\
Pl.foram. & 0.0 & 0.0 & -0.001 & -0.01 \\
\hline
\end{tabular}

tions, and inflowing water and sediment input (Fig. 8).

\section{Carbonate deposits}

Regarding to carbonate deposits, the experiment results show a coherent distribution according to the parameters defined for carbonate production organisms associations (Fig. 9). Thus, a zonation as a function of water depth can be observed from corals placed in the northern area of the basin, benthic foraminifera spread on the whole basin but mainly concentrated in the central part, followed by rhodoliths and planktonic foraminifera in the southern part of the basin.

The complex interaction among the modeled parameters that control the species association evolution and its carbonate production is difficult to analyse. Nevertheless, a detailed study can be done in order to compare the expected and the obtained results. For example, and focused on coralline association, the resulting carbonate distribution and the defined environmental factors (Table 3, section 4.2) can be compared in different time steps (6000y and 11000y are compared in Fig. 10). Under these conditions, the area where corals can live and growth can be delimited by the superposition of each environmental factor. During this period (from 6000 to 11000 years) a marine trangression is modeled, thus the resulting optimum area due to water depth changes according to the evolution of the sea-level position through time. The high slope of the delta front sited in the NE inhibits the development of coral species association in this area. The flow velocity restricts the development of coralline sediment eastwards. Total sediment deposited in each time step is in turn conditioned by the interaction with the other species associations and the available space for deposition.

\section{Facies assemblages}

Results can also be analysed and visualized trough facies assemblages obtained automatically by the program (Fig. 11). Facies are grouped as a function of sediment percentage per each sediment type (obtained from the total sediment deposited) and coloured according to the major sediment every $500 \mathrm{y}$. In this sample experiment, 6 facies assemblages are obtained. Each one is characterized by a mixture of sediments (graphically summarized in Fig. 12), and corresponds with four carbonate-dominated facies (I to IV) and two siliciclastic-dominated facies (V and VI).

Specifically, Facies I is dominated by corals with a contribution larger than $40 \%$; Facies II is dominated by benthic foraminifera with a minimal contribution of $35 \%$; Facies III is characterized mainly by rhodoliths $(>40 \%)$ and planktonic foraminifera $(\sim 40 \%)$; Facies IV is dominated by planktonic foraminifera with a proportion larger than $40 \%$; Facies $\mathrm{V}$ is dominated by coarse siliciclastic sediment $(>40 \%)$; and facies VI is dominated by the finest clastic sediment, with a minimum proportion of $30 \%$.

Additionally, the program can extract a synthetic 1D column at a defined point of the basin (Fig. 11, 1 and 2) representing the sediment deposited, and the corresponding sediment percentage in vertical direction.

\section{Sequential stratigraphy}

From the sea-level variation and the facies assemblage distribution, 9 differentiated genetic types of deposit (systems tracts) belonging to three distinct depositional sequences (A, B, and C) can be interpreted (Fig. 13). The Depositional Sequence A (DSA) is composed of Transgressive (T), Highstand $(\mathrm{H})$ and Forced Regressive (FR) deposits. Depositional Sequence B (DSB) includes a Lowstand (L), Transgressive (T), Highstand $(\mathrm{H})$, and Forced Regressive (FR) genetic units. Depositional Sequence C (DSC) comprises a Lowstand (L) genetic unit followed by Transgressive (T) deposits. Depositional sequences are mainly developed on distallysteepened ramps or in a river delta around the siliciclastic sediment input in the NE part of the basin.

The $\mathrm{T}$ deposits of DSA and DSC (Fig. 13B and D) are stacked in a retrograding pattern (Fig. 14A, B, and 


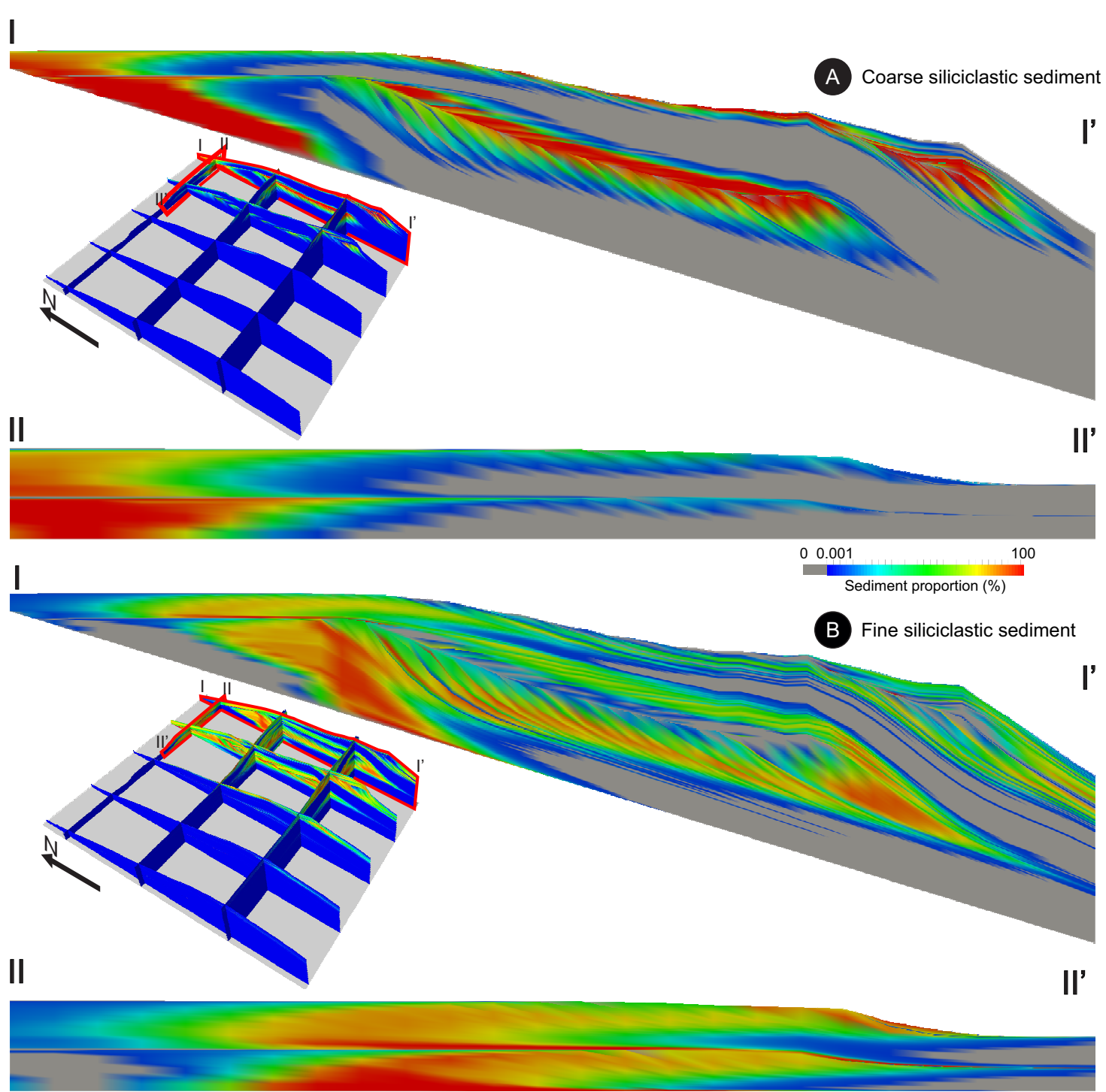

Fig. 8 Distribution in \% at the end of the simulation time for the coarse (A) and fine (B) siliciclastic sediments. Detailed longitudinal and perpendicular cross-sections with a grey mask for values below $0.001 \%$ are amplified for a better comprehension. Vertical exaggeration 10x.

C) and are formed by facies assemblage I in the inner and middle ramp, facies assemblages II, III, and IV in the middle and outer ramp. The facies assemblage $\mathrm{V}$ and VI are also present in the area around the siliciclastic sediment input in the NE of the basin. The T deposits of DSB follow the same pattern as DSA and DSC but facies assemblage II is not present (Fig. 14A).

The $\mathrm{H}$ deposits of DSA and DSB (Fig. 13B and D) exhibit a thin carbonate unit stacked in an aggrading pattern (Fig. 14A and B). The $\mathrm{H}$ genetic type of deposit in DSA is made up of facies assemblage I, which change basinwards to facies assemblages II, III, and IV on the SW carbonate ramp, and facies assemblages V and VI in the NE river delta. In DSB, the facies assemblage II is not present.
The FR deposits in both sequences A and B, correspond to a large river delta system stacked in a prograding pattern (Fig. 13C and 14C, D and E). Similar to the $\mathrm{H}$ units, the $\mathrm{L}$ units are constituted by proximal facies (facies assemblage I), which change basinwards to facies assemblage II. The thickness of these units are thin and the units aggrade.

\subsection{Comparison}

The form, the bathymetry and extension of the theoretical basin are arbitrary and are therefore not comparable with real geological examples. The parameter values of the species associations are taken from the bibliography and the interaction coefficients were es- 

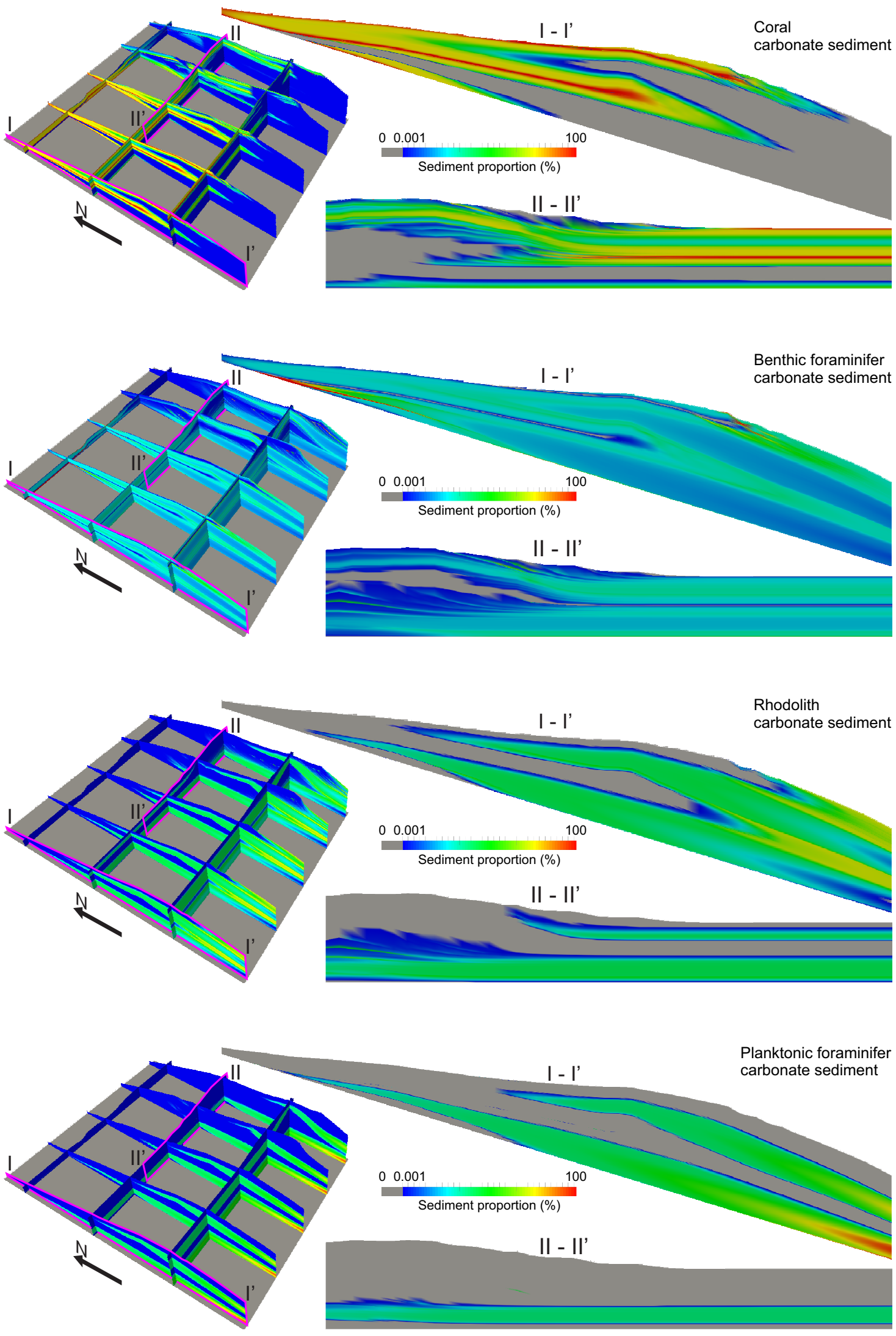

Fig. 9 Carbonate sediment distribution in the basin. Representation for the 4 species associations in proportion of sediment (in $\%$ ). Detailed longitudinal and perpendicular cross-sections with a grey mask for values below $0.001 \%$ are amplified for a better comprehension. Proportions are calculated considering the system: coralline, rhodolith, benthic foraminiferous, planktonic foraminiferous, and coarse and fine siliciclastic sediment. Vertical scale exaggeration 10x. 

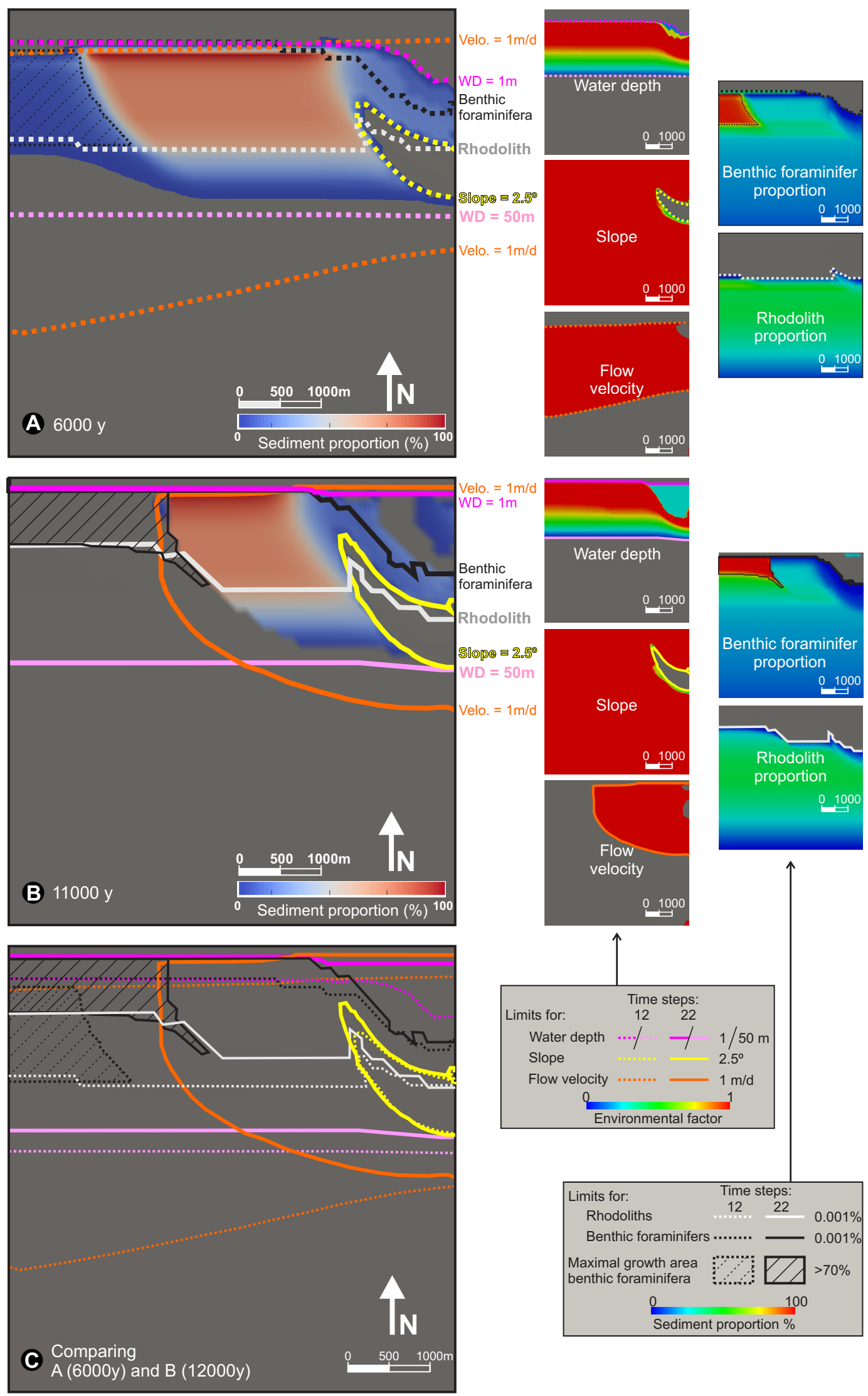

Fig. 10 Coral carbonate sediment distribution in the basin at 6000y (A) and 11000y (B). Environmental factors for coral development are also displayed: water depth (bounded by magenta lines), flow velocity (bounded by orange lines), and slope (bounded by yellow lines). The benthic foraminifera (black lines) and rhodolith (white lines) proportions are also displayed showing the interaction between these species associations and coral carbonate production. The evolution from $\mathrm{A}$ to $\mathrm{B}$ of the environmental parameters is shown in the data comparison $(\mathrm{C})$. 


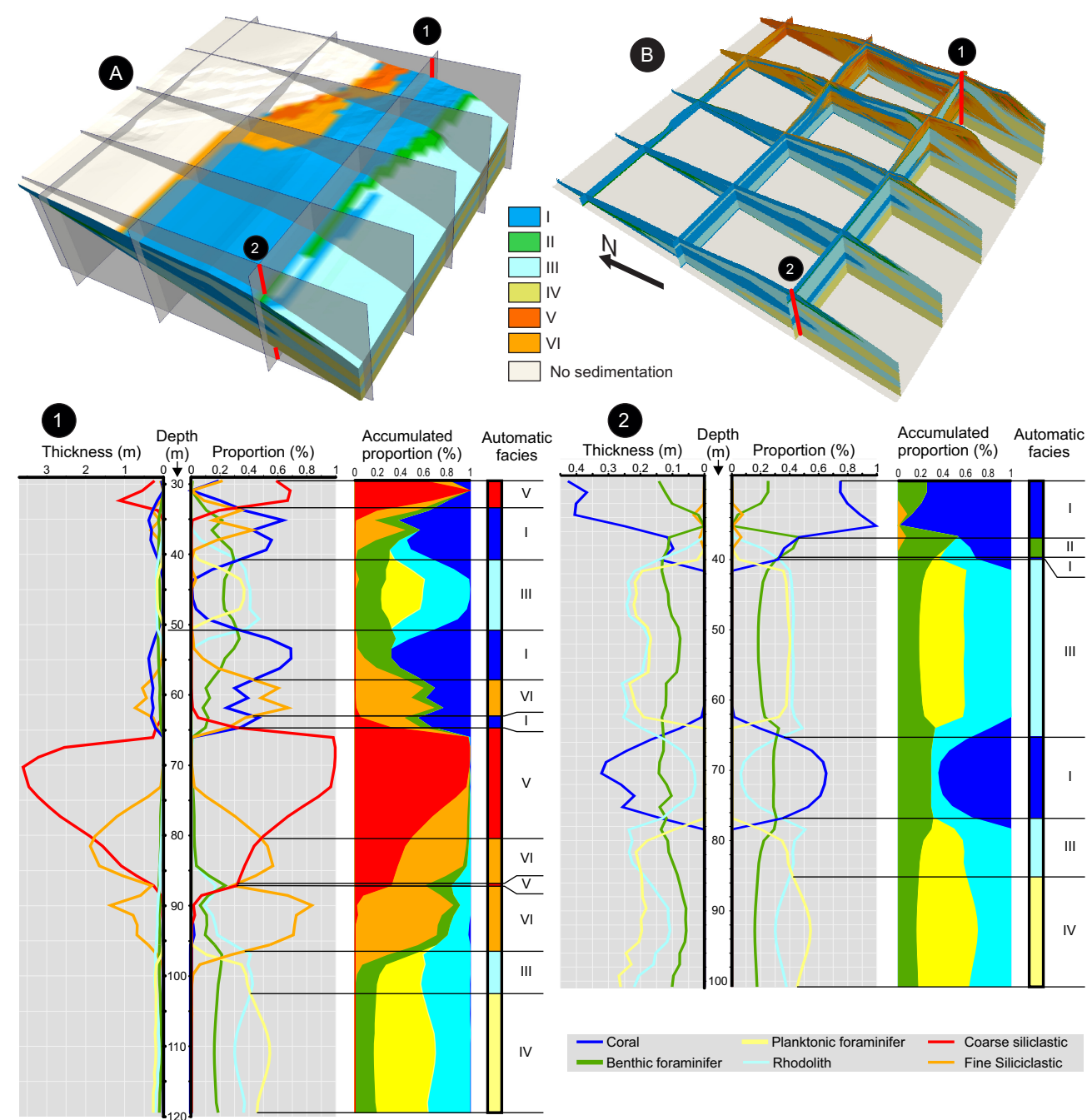

Fig. 11 Facies assemblage representation. A. 3D view and well positions. B. 3D fence diagram. 1D column representation of facies, sediment thickness, sediment proportion, and accumulated proportion at 2 different basin positions, one siliciclastic dominated in the eastern part (1), and other carbonate dominated in the western part (2). Note the 2 main cicles defined in the eustatic curve and revealed in the sedimentary record in both columns.

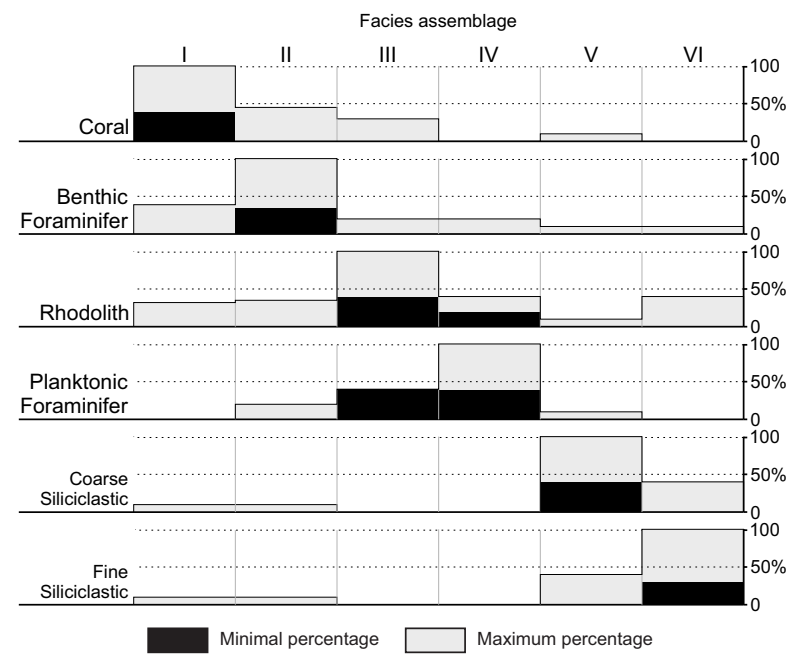

Fig. 12 Proportion of sediment types for each automatic facies assemblage. timated (section 4.2). Therefore, results obtained can only be compared with real carbonate ramps on the basis of the obtained facies distribution.

The species associations modeled in this theoretical example are present in carbonate successions of Oligocene-Miocene age, such as the Asmari Formation in SW Iran [1] and the Ragusa platform in Italy [38].

\section{Asmari Formation:}

The Asmari Formation mainly consists of limestones, dolomitic limestones, and clay-rich limestones. It corresponds to a carbonate platform developed across the Zagros Basin.

According to [1], in the inner ramp, the most abundant skeletal components are larger foraminifera. The presence of porcellanous foraminifera indicates a lowenergy, upper photic, inner depositional environment. 


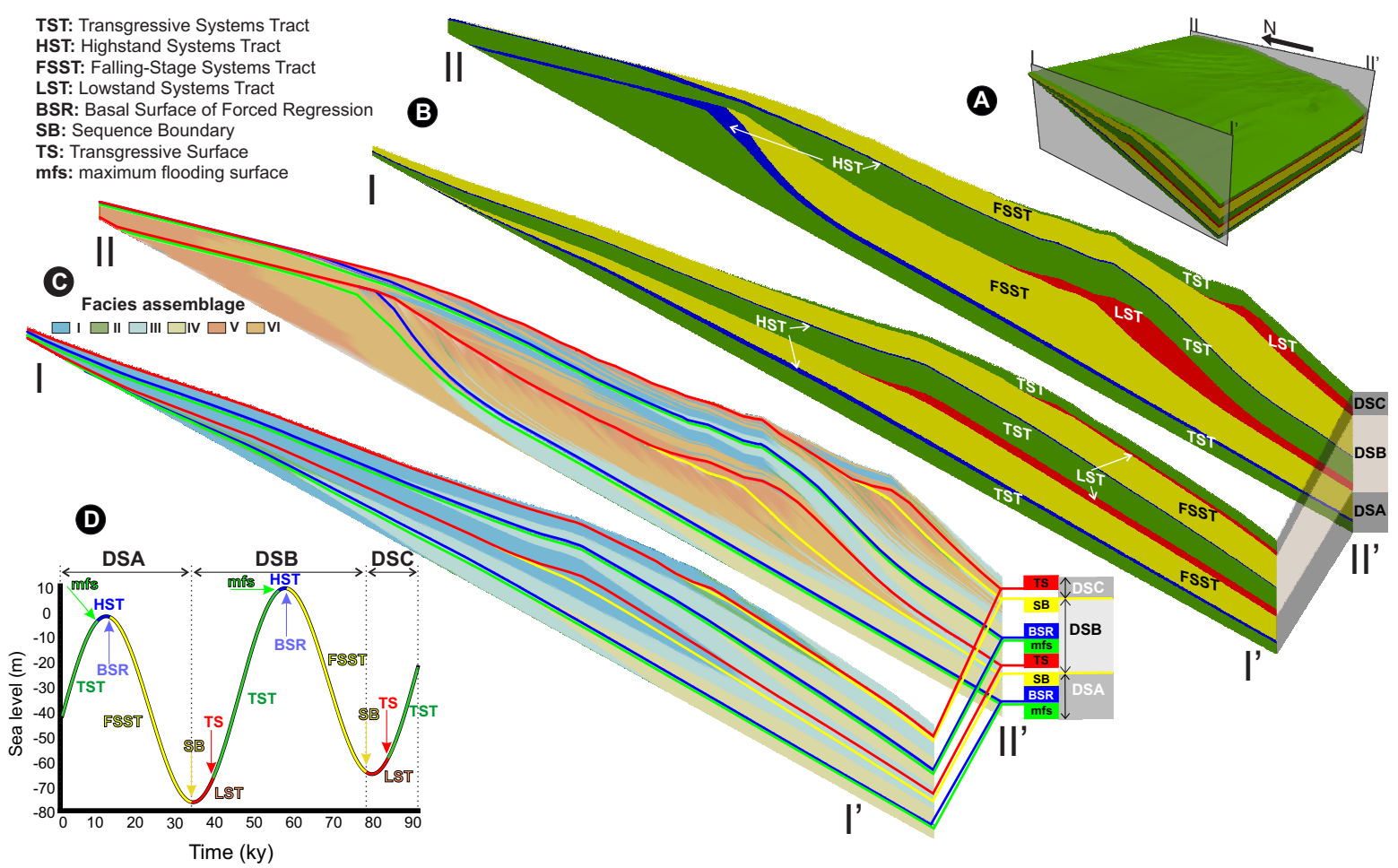

Fig. 13 3D view (A) and cross-sections showing the system tracks (B) and facies assemblages (C) obtained from the sequence stratigraphy analysis, and the defined eustatic curve (D).

The middle ramp deposits are characterized by larger foraminifera with perforate walls indicating a depositional environment situated in the mesophotic to oligophotic zone. The lower photic zone is dominated by large, flat, and perforated foraminifera associated with symbiontbearing diatoms. Lower slope facies are differentiated from upper slope by the greater amount of micritic matrix, an increase in the flatness, and size of the perforate foraminifera and presence of planktonic foraminifera. The outer ramp was characterized by low energy conditions and sedimentation of mudstones with planktonic foraminifera, which indicate deeper water.

\section{The Ragusa ramp:}

Located in SE Sicily, the Ragusa platform corresponds to the outcropping portion of the Hyblean Plateau [38]. Following these authors, the inner ramp is composed by coral-rich, mudstone/wackestone beds. The innermost facies of the inner shallow-water zone comprises gastropods associated with fragments of Corallinaceae red algae, ostracods and green algae. Shelfward, coral colonies extent associated with benthic foraminifera, serpulids, bivalves, and echinoderms, which appear in the outer shallow-water zone. The muddy sediments of the most restricted part of the inner ramp reflect low energy and euphotic conditions. Trophic resources were low enough for scleractinian corals to grow, suggesting oligo-mesotrophic conditions (low-medium nuictrients concentration). Basinwards, the occurrence of packstones in the outer shallow-water zone supports a relative increase in water energy.

In the middle ramp, sediments mainly consist of corallinaceans (branching shapes and spherical rhodoliths) that are associated with chlorozoan biota (sleractinian corals and red algae). Subordinate biota include bryozoans, serpulids, Vermetidae, and small benthic foraminifera. Basinward, benthic foraminifera, as well as echinoids, and planktonic foraminifera complement the biota. Sediments of the middle ramp were likely deposited in the euphotic-mesophotic zone. The deepest associations of scleractinian corals, Vermetidae and benthic foraminifera suggest euphotic water depths [38].

In the outer ramp, the dominating facies consists of planktonic foraminiferal mudstones and wackestones lacking light-dependent biota.

Comparing the three carbonate ramps (Fig. 15), coral species association is present in the inner and middle ramp with different proportion, but follows the same distribution. Benthic foraminifera are present in the inner and middle ramp, except in the theoretical example where have been extended to the outer ramp. Rhodoliths are mainly present in the middle ramp in the three cases, however they are present in the inner 


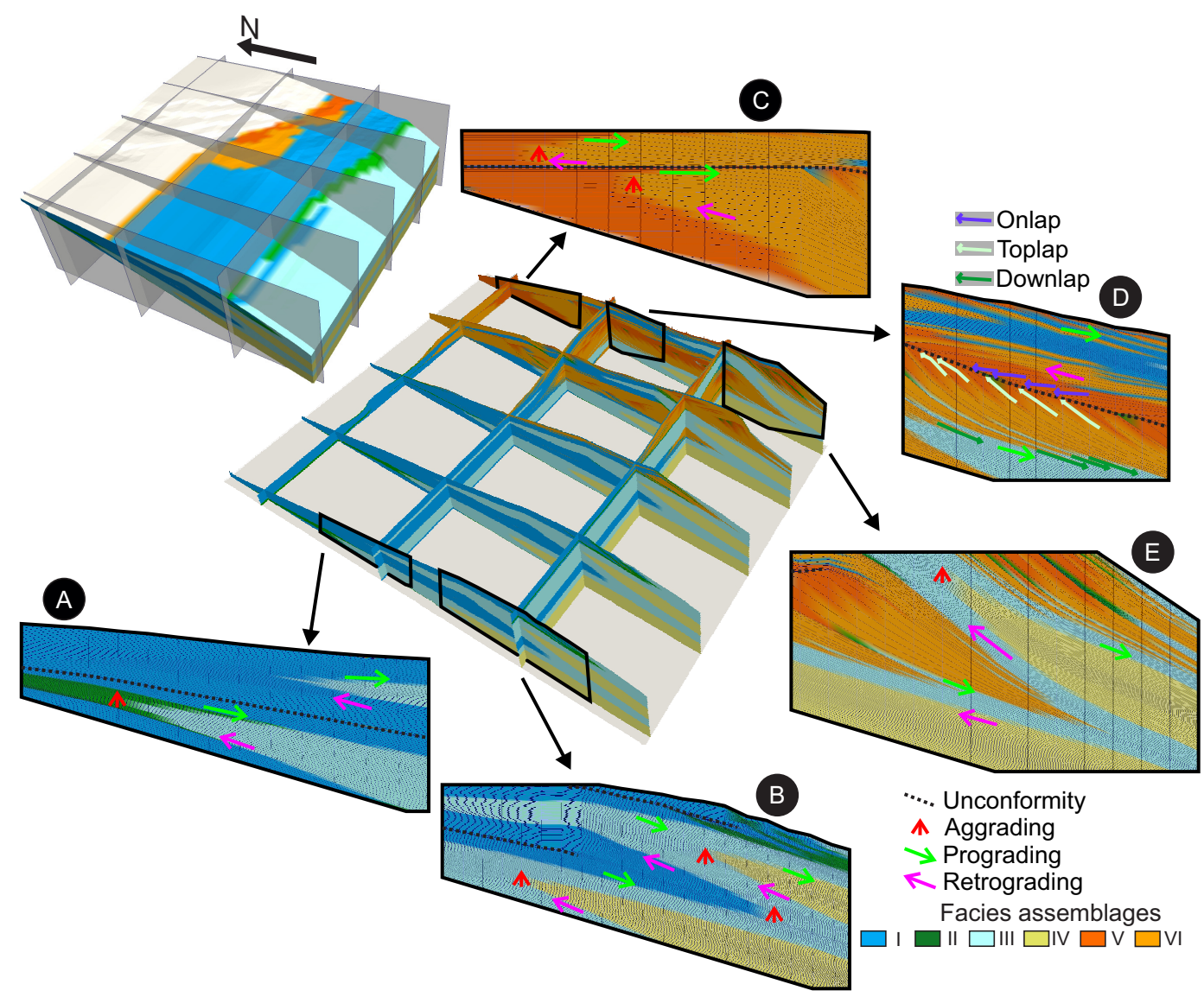

Fig. 14 Patterns present in different sections of the theoretical example. A and B: Enlarged cross-sections with marked patterns in carbonate dominant facies. C, D and E: enlarged cross-sections with marked patterns of siliciclastic dominant facies. Facies assemblages are described in the text. Vertical exaggeration 10x.

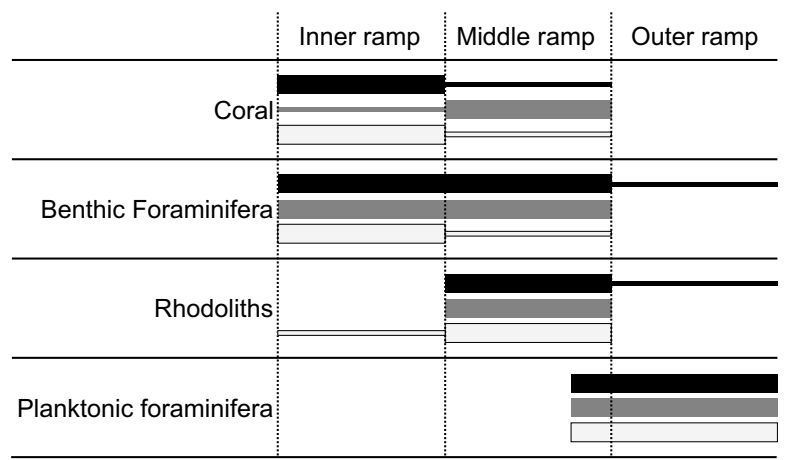

Theoretical example

Asmari Fm. (Amirshahkarami et al., 2007)

Ragusa Platform (Ruchonnet and Kindler, 2010)

Fig. 15 Relative abundances (thick lines represent higher abundance and thin lines represent lower abundance) of carbonate compounds in the compared carbonate ramps.

ramp of Ragusa, and extend to the outer ramp in the theoretical example. Planktonic foraminifera occur in the deepest areas of middle ramp settings and extends to the outer ramp in all examples.
The benthic foraminifera and rhodoliths are present in the outer ramp in the theoretical example, although on a low proportion. These light-dependent biota presence in the outer ramp is the main difference with the real carbonate examples, and it may indicate that the outer ramp is not aphotic in the theoretical example. Thus the theoretical values used and extracted from the bibliography for this kind of species associations differs from the ones in Ragusa and Asmari (that shows also differences between them) indicating probably specific rhodoliths and benthic foraminifera in these platforms.

\section{Conclusions.}

One of the main aspects of SIMSAFADIM-CLASTIC (SF-CL) is the ability to model carbonate production and clastic sedimentation and their interaction, as well as the interplay with the rest of simulated elements. The modeled processes are designed over a geological time at a basin scale using a process-based forward model. This allows the prediction of complex geometries and facies patterns. 
The new model presented for carbonate production illustrates the importance to take into account the biological interactions and intrinsic factors of the carbonate producing organisms (the species growing and the interaction among other species), as well as the environmental parameters, such as energy of the medium, bottom profile, or water depth.

The results of the sample experiment show the potentiality of the code. The example exhibits optimal results for the simulated processes (fluid flow, sediment transport, clastic sedimentation, and carbonate production). From the results obtained, it is possible to see the stratal architecture and stacking patterns of sedimentary bodies and their relationship.

The obtained carbonate production distribution during the modeled time in the basin is a combination of interactions of the species associations with the environmental parameters. The result of these interactions is complex, but some conclusions can be highlighted:

- The slope plays an important role in the delta front in NW part, where most of the clastic sedimentation occurs.

- Due to the initial basin geometry, water depth factor has a great influence in the N-S direction as shown in the facies distribution in vertical and horizontal directions.

- Flow velocity plays an important role in areas near the shoreline combined with the water source, where an important gradient of velocities is present.

- The interaction among species is not clearly visible in the example, despite it is present. The reasons are: (1) the low values taken in the example and (2) interaction do not change in time, but the environmental factors do change, masking this interaction.

Regarding to the comparison with real examples, the facies distribution correlate well based on their position along the ramp. The only exception is in the outer ramp where in the sample experiment presents lightdependent biota, indicating oligophotic conditions, while in the Ragusa and Asmari platforms do not appear. This may indicate that the theoretically lower limit used for rhodoliths and benthic foraminifera and obtained from the bibliography is lower than the expected for the Ragusa and Asmari due to the presence of a specific specie of rhodoliths and benthic foraminifera.

Summing up, we can conclude that the new version of SF-CL is an important step compared with the previous versions, because simulations -such as the example presented herein- would not be possible without the new improvements presented. These improvements condition better the carbonate evolution of the species association, and allow more realistic results since new important parameters can be taken into account.

Acknowledgements This research was carried out in the Geomodels Research Institute (Universitat de Barcelona) and the University of Bayreuth. This work was supported by the projects: INTECTOSAL (CGL2010-21968-C02-01/BTE), MODELGEO (CGL2010-15294) and SALTECRES (CGL2014-54118C2-1-R).

\section{References}

1. Amirshahkarami, M., Vaziri-Moghaddam, H., Taheri, A.: Sedimentary facies and sequence stratigraphy of the Asmari Formation at Chaman-Bolbol, Zagros Basin, Iran. Journal of Asian Earth Sciences 29(56), 947-959 (2007). DOI 10.1016/j.jseaes.2006.06.008

2. Beavington-Penney, S.J., Racey, A.: Ecology of extant nummulitids and other larger benthic foraminifera: applications in palaeoenvironmental analysis. EarthScience Reviews 67(34), 219-265 (2004). DOI 10.1016/j.earscirev.2004.02.005

3. Bice, D.M.: Computer simulation of carbonate platform and basin systems. Kansas Geological Survey Bulletin 233, 431-447 (1991)

4. Bitzer, K., Salas, R.: Simulating Carbonate and Mixed Carbonate-Clastic Sedimentation using Predator-Prey Models. In: D.F. Merriam, J.C. Davis (eds.) Geologic Modeling and Simulation, Computer Applications in the Earth Sciences, pp. 169-204. Springer US (2001)

5. Bitzer, K., Salas, R.: SIMSAFADIM: three-dimensional simulation of stratigraphic architecture and facies distribution modeling of carbonate sediments. Computers \& Geosciences 28(10), 1177-1192 (2002). DOI 10.1016/S0098-3004(02)00037-7

6. Bosence, D., Waltham, D.: Computer modeling the internal architecture of carbonate platforms. Geology 18(1), 26-30 (1990). DOI 10.1130/00917613(1990)018;0026:CMTIAO ¿2.3.CO;2

7. Bosscher, H., Schlager, W.: Computer simulation of reef growth. Sedimentology 39(3), 503-512 (1992). DOI 10.1111/j.1365-3091.1992.tb02130.x

8. Bosscher, H., Southam, J.: CARBPLATA computer model to simulate the development of carbonate platforms. Geology 20(3), 235-238 (1992). DOI 10.1130/0091-7613(1992)020;0235:CACMTS $\_2.3 . C O ; 2$

9. Boylan, A.L., Waltham, D.A., Bosence, D.W.J., Badenas, B., Aurell, M.: Digital rocks: linking forward modelling to carbonate facies. Basin Research 14(3), 401-415 (2002). DOI 10.1046/j.1365-2117.2002.00180.x

10. Burgess, P.M.: CarboCAT: A cellular automata model of heterogeneous carbonate strata. Computers \& Geosciences 53, 129-140 (2013). DOI 10.1016/j.cageo.2011.08.026

11. Burgess, P.M., Emery, D.J.: Sensitive dependence, divergence and unpredictable behaviour in a stratigraphic forward model of a carbonate system. Geological Society, London, Special Publications 239(1), 77-94 (2004). DOI 10.1144/GSL.SP.2004.239.01.06

12. Burgess, P.M., Wright, V.P., Emery, D.: Numerical forward modelling of peritidal carbonate parasequence development: implications for outcrop interpretation. Basin 
Research 13(1), 1-16 (2001). DOI 10.1046/j.13652117.2001.00130.x

13. Carmona, A., Clavera-Gispert, R., Gratacós, O., Hardy, S.: Modelling Syntectonic Sedimentation: Combining a Discrete Element Model of Tectonic Deformation and a Process-based Sedimentary Model in 3d. Mathematical Geosciences 42(5), 519-534 (2010). DOI 10.1007/s11004010-9293-6

14. Chappell, J.: Coral morphology, diversity and reef growth. Nature 286(5770), 249-252 (1980). DOI $10.1038 / 286249 \mathrm{a} 0$

15. Clavera-Gispert, R., Carmona, A., Gratacós, ., TolosanaDelgado, R.: Incorporating nutrients as a limiting factor in carbonate modelling. Palaeogeography, Palaeoclimatology, Palaeoecology 329-330, 150-157 (2012). DOI $10.1016 /$ j.palaeo.2012.02.025

16. Cuevas Castell, J.M., Betzler, C., Rssler, J., Hssner, H., Peinl, M.: Integrating outcrop data and forward computer modelling to unravel the development of a Messinian carbonate platform in SE Spain (Sorbas Basin). Sedimentology 54(2), 423-441 (2007). DOI 10.1111/j.1365-3091.2006.00843.x

17. Demicco, R.V.: CYCOPATH 2da two-dimensional, forward model of cyclic sedimentation on carbonate platforms. Computers \& Geosciences 24(5), 405-423 (1998). DOI 10.1016/S0098-3004(98)00024-7

18. Eppley, R.W.: Temperature and Phytoplankton Growth in the Sea. Fishery Bulletin 70, 1063-1085 (1972)

19. Fay, T.H., Greeff, J.C.: A three species competition model as a decision support tool. Ecological Modelling 211(12), 142-152 (2008). DOI 10.1016/j.ecolmodel.2007.08.023

20. Gillman, M.: An Introduction to Mathematical Models in Ecology and Evolution: Time and Space. John Wiley \& Sons (2009)

21. Granjeon, D., Joseph, P.: Concepts and Applications of a 3-D Multiple Lithology, Diffusive Model in Stratigraphic Modeling. Numerical Experiments in Stratigraphy 62, 197-211 (1999). DOI 10.2110/pec.99.62.0197

22. Gratacós, O., Bitzer, K., Casamor, J.L., Cabrera, L., Calafat, A., Canals, M., Roca, E.: SIMSAFADIMCLASTIC: A new approach to mathematical 3d forward simulation modelling for terrigenous and carbonate marine sedimentation. Geologica Acta 7(3), 311-322 (2009). DOI 10.1344/105.000001390

23. Gratacós, O., Bitzer, K., Casamor, J.L., Cabrera, L., Calafat, A., Canals, M., Roca, E.: Simulating transport and deposition of clastic sediments in an elongate basin using the SIMSAFADIM-CLASTIC program: The Camarasa artificial lake case study (NE Spain). Sedimentary Geology 222(12), 16-26 (2009). DOI 10.1016/j.sedgeo.2009.05.010

24. Hairer, E.: Solving ordinary differential equations I: nonstiff problems, 2nd rev. ed edn. No. 8 in Springer series in computational mathematics. Springer, Heidelberg ; London (2009)

25. Hill, J., Tetzlaff, D., Curtis, A., Wood, R.: Modeling shallow marine carbonate depositional systems. Computers \& Geosciences 35(9), 1862-1874 (2009). DOI $10.1016 /$ j.cageo.2008.12.006

26. Hubbard, D.K., Scaturo, D.: Growth rates of seven species of scleractinean corals from Cane Bay and Salt River, St. Croix, USVI. Bulletin of Marine Science 36(2), 325-338 (1985)

27. Hssner, H., Roessler, J., Betzler, C., Petschick, R., Peinl, M.: Testing 3d computer simulation of carbonate platform growth with REPRO: the Miocene Llucmajor carbonate platform (Mallorca). Palaeogeography, Palaeocli- matology, Palaeoecology 175(14), 239-247 (2001). DOI 10.1016/S0031-0182(01)00374-1

28. Kleypas, J.A., Mcmanus, J.W., Meez, L.a.B.: Environmental Limits to Coral Reef Development: Where Do We Draw the Line? American Zoologist 39(1), 146-159 (1999). DOI 10.1093/icb/39.1.146

29. Letourneur, Y., Ruitton, S., Sartoretto, S.: Environmental and benthic habitat factors structuring the spatial distribution of a summer infralittoral fish assemblage in the north-western Mediterranean Sea. Journal of the Marine Biological Association of the United Kingdom 83(01), 193-204 (2003). DOI 10.1017/S0025315403006970h

30. Morse, J.W., Gledhill, D.K., Millero, F.J.: Caco3 precipitation kinetics in waters from the great Bahama bank: Implications for the relationship between bank hydrochemistry and whitings. Geochimica et Cosmochimica Acta 67(15), 2819-2826 (2003). DOI 10.1016/S00167037(03)00103-0

31. Morse, J.W., Mackenzie, F.T.: Geochemistry of Sedimentary Carbonates. Elsevier (1990)

32. Nordlund, U.: FUZZIM: forward stratigraphic modeling made simple. Computers \& Geosciences 25(4), 449-456 (1999). DOI 10.1016/S0098-3004(98)00151-4

33. Pastor, J.: Mathematical ecology of populations and ecosystems. Wiley-Blackwell Pub. (2008)

34. Paterson, R.J., Whitaker, F.F., Jones, G.D., Smart, P.L., Waltham, D., Felce, G.: Accommodation and Sedimentary Architecture of Isolated Icehouse Carbonate Platforms: Insights from Forward Modeling with CARB3d+. Journal of Sedimentary Research 76(10), 1162-1182 (2006). DOI 10.2110/jsr.2006.113

35. Read, J.F., Osleger, D., Elrick, M.: Two-dimensional modeling of carbonate ramp sequences and component cycles. Kansas Geological Survey Bulletin 233, 473-488 (1991)

36. Roberts, A.: The stability of a feasible random ecosystem. Nature 251(5476), 607-608 (1974). DOI $10.1038 / 251607 \mathrm{a} 0$

37. Roff, J.C., Taylor, M.E., Laughren, J.: Geophysical approaches to the classification, delineation and monitoring of marine habitats and their communities. Aquatic Conservation: Marine and Freshwater Ecosystems 13(1), 77-90 (2003). DOI 10.1002/aqc.525

38. Ruchonnet, C., Kindler, P.: Facies Models and Geometries of the Ragusa Platform (SE Sicily, Italy) Near the SerravallianTortonian Boundary. In: r. Mutti, W. Piller, C. Betzler (eds.) Carbonate Systems during the OligoceneMiocene Climatic Transition, pp. 71-88. WileyBlackwell (2012)

39. Schlager, W.: Carbonate Sedimentology and Sequence Stratigraphy. No. 8 in Concepts in Sedimentology and Paleontology (CSP) Series. SEPM Soc for Sed Geology (2005)

40. Shatalov, M., Greeff, J., Joubert, S., Fedotov, I.: Parametric identification of the model with one predator and two prey species. pp. 101-109 (2008)

41. Steefel, C., MacQuarrie, K.: Approaches to modeling of reactive transport in porous media. In: P. Lichtner, C. Steefel, E. Oelkers (eds.) Reactive transport in porous media, Reviews in Mineralogy, vol. 34, p. 85129 (1996)

42. Steller, D.L., Foster, M.S.: Environmental factors influencing distribution and morphology of rhodoliths in Baha Concepcin, B.C.S., Mxico. Journal of Experimental Marine Biology and Ecology 194(2), 201-212 (1995). DOI 10.1016/0022-0981(95)00086-0

43. Tregonning, K., Roberts, A.: Complex systems which evolve towards homeostasis. Nature 281(5732), 563-564 (1979). DOI 10.1038/281563a0 
44. Warrlich, G.M.D., Waltham, D.A., Bosence, D.W.J.: Quantifying the sequence stratigraphy and drowning mechanisms of atolls using a new 3-D forward stratigraphic modelling program (CARBONATE 3d). Basin Research 14(3), 379-400 (2002). DOI 10.1046/j.13652117.2002.00181.x

45. Westphal, H., Riegl, B., Eberli, G.P.: Carbonate Depositional Systems: Assessing Dimensions and Controlling Parameters: The Bahamas, Belize and the Persian/Arabian Gulf. Springer Science \& Business Media (2010) 